Voix et Images

voixetimages

\title{
Absence de Rina Lasnier
}

\section{André Brochu}

Volume 1, numéro 2, décembre 1975

\section{Raoul Duguay}

URI : https://id.erudit.org/iderudit/290073ar

DOI : https://doi.org/10.7202/290073ar

Aller au sommaire du numéro

Éditeur(s)

Les Presses de l'Université du Québec

ISSN

0318-9201 (imprimé)

1705-933X (numérique)

Découvrir la revue

Citer cet article

Brochu, A. (1975). Absence de Rina Lasnier. Voix et Images, 1(2), 173-181.

https://doi.org/10.7202/290073ar

Ce document est protégé par la loi sur le droit d'auteur. L'utilisation des services d'Érudit (y compris la reproduction) est assujettie à sa politique d'utilisation que vous pouvez consulter en ligne.

https://apropos.erudit.org/fr/usagers/politique-dutilisation/
Cet article est diffusé et préservé par Érudit.

Érudit est un consortium interuniversitaire sans but lucratif composé de l’Université de Montréal, l'Université Laval et l'Université du Québec à Montréal. Il a pour mission la promotion et la valorisation de la recherche. https://www.erudit.org/fr/ 


\section{Absence de Rina Lasnier}

Pour un de mes amis, grand amateur de paradoxes, Rina Lasnier est l'exemple même de quelqu'un qui écrit mal, qui bourre son langage de métaphores hasardeuses dans le seul but de "faire poétique", qui accumule les redondances sémantiques et n'a pas le sens du mot juste. Et mon ami n'aurait pas tort s'il n'oubliait, hélas, d'interroger d'un peu plus près le poème et d'y chercher la raison nécessaire de cette surcharge, ce tropplein de l'écriture qui n'en masquerait d'après lui que le vide.

Un poème répond fort bien à ses objections. C'est " Jungle de feuilles ${ }^{1}$ " qu'on peut lire, avec un peu d'audace, comme un art poétique. Le premier vers, "Absence de la forêt suffoquée de feuilles", pourrait se traduire ainsi: vide du poème trop chargé d'images. Très souvent, la poésie de Rina Lasnier se lit comme une allégorie, mais dont le terme symbolisé non seulement reste implicite, mais peut être plusieurs. "Jungle de feuilles" est une énorme synecdoque particularisante du trop-plein qui équivaut au néant, d'une présence qui n'est qu'absence, et par là, il peut devenir la métaphore de tout ce qui particularise cette idée ${ }^{2}$ : il peut donc être la métaphore d'une congestion de métaphores.

La congestion métaphorique, notons-le, est une des tendances de la poésie moderne, du moins de celle qui s'est vouée à l'exploration, sinon à l'exploitation de l'imaginaire. Denis Roche, dans "La Poésie est inadmissible d'ailleurs elle n'existe pas ${ }^{3}$ ", condamne cette «intelligence SYMBOLARDE de la poésie dont l'écriture n'a jamais été vue que comme puérilement évocatrice d'une activité personnelle esthétisante (nous parlons ici d'une esthétique sociale/morale) ". Ce faisant, il indique bien tout ce qu'une poésie moderne, humaniste et imagée, doit au symbolisme d'antan, dont elle a seulement complexifié la poétique en disjoignant le symbole de son référent (lequel, du coup, devenait pluriel). Mais une pratique du langage fon-

1. Rina Lasnier, Poèmes I, Montréal, Fides, «Nénuphar », 1972, p. 273.

2. La métaphore, comme le montrent les auteurs de Rhétorique générale, Paris, Larousse, 1970, est une double synecdoque: le plus souvent une synecdoque généralisante, prolongée en la synecdoque inverse. On lira aussi, si l'on peut, mon "Ebauche d'une rhétorique de "la Malemer", dans la Voix des poètes, Paris, coll. Les Pharaons, $n^{\circ} 55$, automne 1974, p. $42-53$.

3. Théorie d'ensemble, par le groupe Tel Quel, Paris, Seuil, 1968, p. 221-233. 
dée sur l'exploitation des seules figures syntaxiques, comme l'est le formalisme d'un Denis Roche ou d'un Pleynet, n'est pas forcément plus «poétique" que l'autre, et on comprend du reste que l'auteur du Mécrit en vienne à juger toute poésie inadmissible, voire inexistante.

Lisons, maintenant, tout «Jungle de feuilles» et voyons ce qu'il advient d'un trop qui rime avec rien.

11 Absence de la forêt suffoquée de feuilles,

2 luxuriance à pourrir l'armature de l'arbre,

3 toison sans tête, sans ossature, monstre de pelage;

4 frondaisons sans sursis de ciel aux bras des branches.

II 1 Chaque feuille grasse collée à la graisse de la moiteur,

2 enchevêtrement poisseux de tous les rampements mous,

3 chair de chair sans retentissement de cris et de flèches:

4 marécage moisissant le socle de la cité.

III 1 Labyrinthe des léthargies sans fuite de rêve,

2 nuit verdâtre sans plumage d'étoiles, sans corne de lune brameuse.

IV 1 Ramas de feuilles refusant d'essuyer les pieds de la pluie,

2 chaque feuille refusant l'assaut de la pluie.et du vent,

3 chaque feuille serait note de pluie, hymne de vent;

4 chaque arbre serait l'armature du feu futur.

$\checkmark 1$ Fermentation sans fruit entamé de faim fiévreuse,

2 stérile gravitation de l'identité sans issue de mort.

3 La forêt marcherait vers un seul apeurement d'eau,

4 la forêt s'évaserait pour une seule coulée d'oiseaux,

5 la forêt s'ordonnerait autour du péril de l'étincelle;

6 jungle tiède où manque la gloire amoureuse d'un corps incendié.

Cette accumulation de notations, qui sont autant de modulations de l'idée du premier vers, constitue une vaste expolition, selon la définition qu'en donne Fontanier ${ }^{4}$. En elle, il y a de l'hystérie. La prolixité de l'invention, l'orgie d'images, la largeur du rythme, la densité étouffante de la phrase sont autant de manifestations d'une harmonie entre le langage et le vécu représenté. La jungle est jungle de mots et, dans son espace carré, elle dispose ses menaces: partout des rampements mous, des labyrinthes, la prolifération végétale absolue.

Partout l'inceste.

Non pas celui de l'enfant et de la mère mais l'autre, Origine de toute faute: celui de la mère avec elle-même, en plénitude d'abjection.

Car la mère, quand elle refuse l'enfant, ne peut se donner qu'à soimême. C'est ainsi que, présente absolument à soi, elle est absence. Absence à l'autre d'abord, l'homme ou l'enfant qui serait ce trou en elle qui la ferait histoire, temps en marche vers une rédemption.

Regardons-y de plus près et cherchons à épouser (peut-on le faire sans souffrance?) le mouvement du texte se mettant au monde, à la fois matrice ("forme») et messager («contenu»). Émissaire du Verbe, qui est l'auteur.

4. L'expolition est la répétition «d'une mème pensée sous différents aspects ou sous différents tours, afin de la rendre plus sensible ou plus intéressante". Les Figures du discours, Paris, Flammarion, 1968, p. 420. 


\section{D'UN RÉCIT ENRAYÉ}

Tout commence par une suffocation $(1,1)$, état de fait ponctuel qui sépare la vie qui respire du resserrement brutal de la mort. La suffocation, état critique c'est-à-dire de crise, est le résultat d'un long processus. Les arbres ont peu à peu occupé tout l'espace de leur vitalité, épuisé leurs possibilités objectives, légitimes d'expansion. Ils n'ont pas épuisé pour autant leurs virtualités de croissance, et c'est là que commence le drame. Ne pouvant plus s'étendre à l'extérieur, ils se gêneront à l'intérieur, dépasseront leur mesure normale de compacité, s'engorgeront d'eux-mêmes. L'«absence " de cette forêt suffoquée est précisément celle du désir, son objet ne pouvant plus être que la mort.

Petite digression. Rina Lasnier, qui a lu Racine, sait que les serpents sifflent sur nos têtes, mais que la forêt suffoque de feuilles: soit dit pour la tant décriée harmonie imitative.

Elle sait, du reste, que la rime peut se faire au début du vers (absence - luxuriance; toison-frondaisons) et que cela convient très bien à sa forêt baroque, véritable monde à l'envers - ou monde en marche vers l'envers, en régression vers son néant.

Le premier vers porte un diagnostic métaphysique: la forêt suffoque, voilà pourquoi cette matière est absence. Le deuxième examine de plus près les causes: la luxuriance (n'est propre qu') à pourrir l'armature de l'arbre, à miner toute verticalité. C'est bien en quoi elle ressemble à la luxure, qui est fatale au «figuier maudit» 5 . Le gaspil de la pulsion (sans frein ni frayage) est cause d'entropie. Son équivalent collectif est la croissance démesurée, exponentielle dans un monde clos, dont nous entretenait récemment la revue Critère ${ }^{6}$. Toute éjaculation contient la loi de son arrêt.

Imaginons maintenant la personnification animale de cette luxuriance, être réduit à la seule faculté de se transmettre, comme les organismes unicellulaires, ou encore un peuple de moutons voué à la stricte survie biologique... Nous avons alors cette métonymique «toison sans tête, sans ossature, monstre de pelage» $(1,3)$. A la forêt, il manque la faculté de limiter le corps par l'idée, de convertir en énergie pensante la pulsion vitale. "Toison sans tête " contient un chiasme phonétique $(\mathrm{t}-$ /sifflante + voyelle nasale/ - /sifflante + voyelle nasale/ $-t$ ) qui suggère bien la circularité d'un corps fermé. "Monstre de pelage": synecdoque superbe, où le référent (pelage) est devenu l'épithète de son attribut substantivé (monstrueux $\rightarrow$ monstre), cette créature de fable devenant à son tour métaphore de la forêt.

Mais ne nous égarons pas dans la jungle des figures: l'analyse y contracterait quelque fièvre fatale, du moins pour la lecture.

5. Dans "le Figuier maudit" (Escales, 1950), c'est effectivement la luxure qui pourrit l'arbre. Voix et images du pays, ancienne manière, a publié une excellente analyse du "Figuier maudit", sous la signature de Chanel Malenfant (VIPIX, 1975, p. 113-138).

6. J'ai fait le compte rendu de ce numéro, intitulé Croissance et démesure, dans la précédente livraison de Voix et images. 
La trop grande luxuriance causait la suffocation, semblable à celle d'un animal plein, entropique ("sans ossature"), sans tête pour respirer: viande au service du pelage. Le diagnostic se porte ensuite vers le manque, qui est d'ordre "pneumatique " ou, soyons braves, spirituel: "frondaisons sans sursis de ciel aux bras des branches". Les mains trop pleines (les frondaisons) ne peuvent accueillir le ciel dont elles ont besoin et qui leur permettrait de surseoir à la mort. L'absence de sursis répète, dans l'ordre du temps, la carence de l'espace vital.

L'assimilation des branches à des bras fait plus que suggérer une personnification de la forêt en quelque foule massive et parquée (pensons aux auditoires d'un führer, ou aux cent députés de l'Assemblée nationale). Elle connote, en accord avec l'inspiration du poète, le bras nu d'un Dieu sur la traverse de la Croix. Jésus n'est-il pas ce sursis de ciel accordé à la forêt des hommes, ce trou d'azur par quoi la matière trouve un centre et un sens? En termes sartriens, il serait le pour-soi logé au cœur de l'en-soi du monde. Qu'on croie ou non à la fable de Dieu, on peut penser, rien en tout cas ne m'en empêche, à la conscience ruptive et à la mesure qu'elle institue, ou devrait instaurer, au sein de la nature.

La matière sans conscience ressemble à nos eaux sans poissons, à nos villes sans maires; au Québec actuel, charogne repue de son innombrable impuissance.

Toute cette “démagogie " littéraire fait sens, bien entendu, en dehors du poème. Mais le poème ne fait pas sens en dehors du monde. Ou de moi. Ou de quiconque le lit. Je ne puis, lisant “toison sans tête", m'empêcher de penser à quelque ministre bien coiffé. Le rire permet d'oublier notre détresse.

Le poème est la trace d'une existence faite langage, par l'autorité du poète. Et c'est cette autorité qui le destine à la lecture personnelle, laquelle n'est pas pure et simple consommation de formes.

Pour résumer la première strophe, je dirai que toute suffocation demande apaisement mais que le remède, ici, est absent. Cette situation est fortement narrative: face au malade qui étouffe, on ne tient pas en place; on voudrait que la crise aboutisse. Un dénouement est nécessaire et, avec lui, une transformation c'est-à-dire, en fin de compte, une " histoire».

Or Rina Lasnier, par une extraordinaire habileté, refuse la narration. C'est que la trop-présence ne ménage en elle aucune place pour le dégorgement narratif. La suffocation, situation ponctuelle, restera situation; la description, qui n'est nullement un parcours mais la répétition variée $\mathrm{du}$ même état de fait, est essentiellement ici récit enrayé, pellicule en panne dans le rayon du projecteur. C'est l'extrême tension créée par l'immobilité de ce qui doit être mobile, qui ouvre l'espace de tout le poème et assure la dynamique de sa lecture. On passera du premier vers au dernier en restant sous le coup d'un arrêt, d'un souffle précisément coupé. À ce moment, le poème est trop plein (de lui-même), il mime la matrice étouffante dont voudrait se dégager le verbe enfant pour prendre sa course et hurler sa liberté. Une extrême violence couve sous l'entassement des phrases 
nominales et c'est celle, que nous saluerons plus bas, du verbe conditionnel qui remplira optativement le manque suggéré dès la première strophe, par ce qu'on pourrait appeler les adjectifs privatifs («sans sursis de ciel aux bras des branches» est l'équivalent syntaxique de «non aérées»; «sans ossature", de «non ossuée", etc.).

Tout le poème cherchera à préciser le diagnostic initial, et il le fera, comme le fait peut-être toute écriture, en remontant au tuf originel qui est, pour la psychanalyse, l'cEdipe ou l'inconscient et qui est encore, pour le révolutionnaire - toujours à l'affût! - ce que Berque appelle le fondamental.

\section{D'UNE REDONDANCE TROUBLANTE}

La première strophe nous conduisait à l'orée de la forêt compacte, nous mettait le nez sur son trop-plein, toison démente et qui pourrit l'arbre (l'érection).

La deuxième nous plonge dedans.

Dedans le gras.

La sémantique de la première strophe respirait un peu, grâce au solide oxymore du premier vers. C'est la redondance qui, maintenant, nous accueille. Les feuilles grasses sont collées à la graisse de la moiteur, qui est sans doute moiteur de l'air, mais qu'est-ce que la graisse de la moiteur de l'air sinon la moiteur elle-même? ou le gras des feuilles?

Je ne connais guère d'équivalent, dans la poésie française, de cette structure tropologique, du moins depuis Ronsard que les pléonasmes n'effrayaient pas. Je cite deux vers de son plus beau poème:

\section{Quand l'Aube de ses pleurs au poinct du jour l'arrose}

(où on peut, à la rigueur, considérer que l'Aube est une divinité; mais l'imagine-t-on arrosant ses jardins en plein midi?)

et:

\section{Embaumant les jardins et les arbres d'odeur ${ }^{7}$}

(de quoi d'autre peut-on embaumer quelque chose?)

Les redondances, par contre, abondent dans notre langue parlée et notre langue écrite, témoignant sans doute d'un fonds germinatif de la conscience locutrice qui n'existe plus en France depuis le Grand Siècle, Malherbe ayant su cultiver son lecteur.

Voilà donc, chez Rina Lasnier, un élément propre à choquer une oreille d'outre-mer. Cependant rien ne dit que de semblables redondances ne puissent être perçues comme de véritables et "saines" audaces linguistiques dans une conception renouvelée et élargie de la poétique française.

Ce que suggère tout ce tas de feuilles grasses, littéralement mêlées à l'air gras, c'est une lubrification des surfaces "sans sursis de ciel ", et peut-

7. Ronsard, les Amours de Marie, "Comme on voit sur la branche au mois de may la rose". 
être une exultation auto-érotique. Passée la «toison» $(1,3)$, voici peut-être la vulve, où ombre et chair se confondent. Plus loin viendra le "labyrinthe» vaginal $(I I, 1)$.

Le vers suivant $(11,2)$ évoque un «enchevêtrement poisseux de tous les rampements mous"; ici, une symbolique sexuelle masculine est présentée sous une forme éminemment dégradée, voire invertie, celle d'un nœud pénien (cf. nœud de vipères) qui s'oppose au aretentissement de cris et de flèches" - inexistant - du vers 3. Mais l'antithèse est vite suivie d'une redondance, bien affichée celle-là. "Chair de chair» désigne une quintessence, un noyau de substance où la matière est absolument impropre à aucune conversion.

Le dernier vers de la strophe $(11,4)$ rappelle des descriptions de la Voie royale, de Malraux. II met en relation la forêt avec un autre, qui n'est plus le ciel mais la cité. Notons que, graphématiquement, la différence n'est pas si grande. De quelle cité s'agit-il? Il semble qu'elle s'élève à la périphérie de la jungle, qui auprès d'elle fait figure de marécage. On se sent en plein pays tropical. Quoi qu'il en soit, la pauvre subit le même sort que l'arbre (cf. 1,2), que tout ce qui est vertical et solide. C'est la culture dégradée par la nature, forme de pollution qui commence à dater... Sans doute est-elle l'homme, refusé par la mère fermée (mâle-mère), comme l'était le ciel, lui aussi masculin. La redondance sémantique de la strophe suggère bien une féminité pleine d'elle-même et qui n'admet aucune pénétration; au contraire, elle fait plutôt tache d'huile et gagne de nouveaux espaces à la mort.

Entre la redondance (qui prédomine en II) et l'oxymore (qui prédomine en 1), la différence est moins grande qu'on ne pense. Entre le plein qui est vide et le plein qui est plein, une vérité commune se dessine: celle du trop, de l'excès. La redondance n'est pas la négation de l'oxymore mais son corrélat. Ce qu'elle a de troublant, voire de choquant, c'est qu'elle met le même en disjonction avec lui-même, sans faire appel à son contraire. Elle est bien "chair de chair», comme si la chair pouvait être non-chair (mais sans être l'esprit). Elle est l'ébranlement sur place d'une catégorie logique, un impossible "en-pour-soi", bien propre à faire sauter la langue ou la raison. Les schizophrènes seuls, plus Deleuze, peuvent en penser à fond la logique qui n'est même plus celle de la raison dialectique: elle est monologisme sans fin, loi du corps sans organes ${ }^{8}$ ou de la sphère parménidienne.

\section{DU LABYRINTHE}

Nous voici engagés dans un sombre conduit (III,1).

La logique du labyrinthe est d'espacer la redondance. En lui, on peut se déplacer, aller ailleurs, mais on reste toujours ici. Aussi le labyrinthe

8. Cf. Deleuze et Guattari, l'Ant1-CEdipe, Paris, Minuit, 1972. Les auteurs nous y invitent à libérer le processus schizophrénique, seule façon de faire échec au capitalisme. Pourtant mai 68 , schize entre les schizes, n'a pas renversé grand' chose... 
est-il toujours celui des "léthargies", celles-ci comprenant le somnambulisme.

Toute régression nous plonge au cœur d'un faux circuit qui paralyse, que n'éclaire plus même l'issue du rêve. C'est que l'imaginaire est près du jour de la conscience, et plein de sa lumière. C'est au-dessous que s'ouvre le noir tunnel de l'inconscient. On lira Lacan, ou ses traducteurs, làdessus. Le symbolique est, d'une certaine façon, plus fondamental que l'imaginaire puisqu'il structure tout l'inconscient. Le psychotique se débat bien au-dessous de ses fantasmes, dans le ça-parle originaire dont un signifiant est forclos: ne serait-ce pas, toujours, le Nom-du-père?

Ici se tait, précisément, le Minotaure, qu'on peut imaginer, comme certaines divinités égyptiennes, recouvert de plumes («nuit verdâtre sans plumage d'étoiles»), et, plus naturellement, doté d'une tête bovine («sans corne de lune brameuse $)$.

Bien entendu, on peut lire ces vers dans une perspective plus réaliste: la forêt, lieu où l'on se perd et où ne parvient pas la lumière, ni celle des étoiles, ni celle de la lune, est dépourvue d'oiseaux et de bêtes (orignaux), de même qu'elle est inhabitée des hommes ("sans retentissement de cris et de flèches", II,3). Mais l'agencement de ces éléments n'a rien de réaliste, et le lecteur peut s'abandonner rêveusement (lui qui peut fuir!) aux sollicitations de son propre désir... de savoir.

Le labyrinthe apparaît dans la strophe médiane (III), la plus courte du poème ( 2 vers). Lui qui est sans commencement ni fin, il est le terme de la plongée au cœur de la jungle. Les strophes suivantes "dé-régresseront" en quelque sorte vers le point de vue initial, nous ramenant aux feuilles (IV) puis à la «forêt" (V).

\section{D'UN SALUT CONDITIONNEL}

Quand, par le verbe poétique, le poète descend au fond de soi y affronter son monstre, toujours maternel ${ }^{9}$ (les cornes du Minotaure n'y changent rien, d'ailleurs il est absent: le monstre, c'est le trou gras), il ne peut, comme Orphée, qu'en remonter - s'il est un vrai poète - mais mutilé de l'image d'Eurydice. C'est la lyre qu'il rapporte des méandres de son cœur, frémissante du chant:

Et j'ai deux fois vainqueur traversé l'Achéron,

Modulant tour à tour sur la lyre d'Orphée

Les soupirs de la sainte et les cris de la fée.

(Nerval, «El Desdichado»)

A travers la statique descriptive de la "Jungle de feuilles", au titre tout redondant, sorte de métonymie in praesentia, c'est bien le drame orphique de l'entrée en soi du poète, de sa plongée toujours recommencée au «ventre de l'eau la plus basse» («la Malemer»), qui se fait entendre et

9. Sur le "monstre maternel", I faut lire les Images de la mère, de Julien Bigras, Saint-Jérôme et Paris, Interprétation et Hachette, 1971. 
qui permet au poète, une fois son désir épuré de l'objet trop humain, de préciser le Manque, par quoi tout le plein est absence.

$\mathrm{Ce}$ Manque, maintenant, prend véritablement forme. D'abord il est manque voulu, c'est-à-dire refus (IV,1). Refus du salut. Marie-Madeleine, la pécheresse, avait oint des huiles les plus précieuses les pieds de Jésus, puis les avait essuyés de ses cheveux - toison non sans tête! La forêt, elle, refuse "d'essuyer les pieds de la pluie", cet émissaire du ciel. Le gras refuse l'eau, qui est séminale, étant la condition de la fertilité. Le gras refuse la fraîcheur et la lustration. Pluie et vent, qui font assaut (IV,2), l'air et l'eau étant l'homme, se heurtent au non de la terre feuillue. Chaque feuille, pourtant, "serait note de pluie, hymne de vent" (IV,3), clavier vibrant du chant céleste. Qu'on ne s'étonne pas de cette trop discrète introduction, dans la syntaxe du texte (ici pure parataxe) du virtuel qui est le salut. Si plein, et en même temps si mou est le refus, que l'antithèse doit être devinée. Les mêmes mots (IV, 2 et 3 ) expriment deux états opposés, rappelant ainsi l'omni-présente Redondance. Seul le temps du verbe, en évoquant un état autre qui est un état contraire, porte le poids de la figure.

Elle qui refuse l'air et l'eau, si elle les accueillait, la forêt-terre pourrait être armature du feu $(I V, 4)$. Voilà un bel exemple de logique poétique dont la justesse échappe au regard trop raisonneur du pur analyste. Comment la pluie peut-elle être le feu? - Par la loi des complémentaires, sans doute. L'arbre, dont l'armature se liquéfiait par la luxuriance, en recevant l'eau retrouverait le feu du ciel. En retrouvant son intégrité ligneuse, il pourrait être porteur de feu, buisson ardent, fête rouge pour le réchauffement des hommes. Telle est la Croix, d'où rayonne Jésus.

Comme le figuier stérile, maudit de Dieu, la forêt est "sans fruit" $(V, 1)$. Plus précisément, elle est fermentation mais ne produit rien qu'ellemême, rien qu'en-soi. Le fruit «entamé de faim fiévreuse" serait l'enfant, et mieux encore l'enfant-Dieu:

Tu nais sans cesse de moi comme les mille bras des vagues courant sur la mer toujours étrangère;

\section{C'est moi ce charroi d'ondes pour mûrir ton destın comme midi au sommet d'une cloche... ${ }^{10}$}

En somme, si la mer peut produire la spirale rédemptrice, la jungle de feuilles est, pour sa part, le symbole d'une luxuriance sans objet, sans but, d'une virginité impure. Elle est Ėve non pardonnée (sans enfant). La mer est maria, nativité du feu ${ }^{11}$ : elle est la Vierge-Mère, oxymore absolu.

Pour que le même soit fécond, puisse donner naissance à l'autre, il faut qu'il accepte la mort. La mort est l'issue de l'identité, comme le rêve était l'issue du labyrinthe (III,1). Cela, Rina Lasnier le dit dans un vers extraordinairement intelligent, bien qu'«intellectuel »:

stérile gravitation de l'identité sans issue de mort

10. "Présence de l'absence", Poèmes I, p. 259.

11. Dernier vers de «la Malemer». Nous le citons infra. 
Il est peu de poètes, chez nous, qui aient approché de si près le ciel ontologique, sans perdre de vue le sol du poème. C'est une des joies que procure la lecture de Lasnier que de trouver chez elle une pensée articulée, mêlée à une imagerie somptueuse, comme chez Hugo. Peut-être faut-il chercher là les véritables racines d'une philosophie québécoise (de même que chez Ernest Gagnon s.j., Vadeboncoeur, Dumont et Grand'maison, ces catholiques!).

Au plan formel, la dernière strophe résume bien l'ensemble du poème. Les deux premiers vers, nous venons de le voir, décrivent la forêt telle qu'elle est, en deux phrases nominales qui contiennent des adjectifs privatifs (sans fruit..., sans issue de mort), rappelant ainsi les trois premières strophes. Les trois suivants (V, 3-5) sont des phrases normales, simples, qui contiennent un verbe au conditionnel, rappelant la strophe IV. Le dernier $(V, 6)$ revient à la structure initiale, mais en verbalisant ("où manque...") la marque privative (sans).

Toutes les strophes sont de quatre vers, sauf la troisième, de deux vers, et la cinquième, de six vers, qui se font équilibre. On a vu que la strophe III nous menait au cœur qui était vide: le labyrinthe; la dernière nous ramène au tout, la forêt, en montrant précisément que le véritable cœur, Centre et Sens, est ailleurs, manque au tout. Et ce cœur serait précisément un trou, une poche d'air et d'eau qui rendrait possible une tendre peur $(V, 3)$ ou une "coulée d'oiseaux" $(V, 4)$, sorte de pluie de plumes (cf. IV,1 et 111,2 ), semence sanctifiante.

L' «apeurement d'eau» vers quoi marcherait la forêt est peut-être celui, commun à la mère et l'enfant, de la distance qui les sépare. L'eau alors n'est plus la pluie qui fait assaut, mais les larmes qui bénissent. L'eau peureuse est pleureuse... surtout si le bonheur est cause de sa peur.

Évasée, ensemencée d'oiseaux, la forêt pourrait alors couver le "péril de l'étincelle» $(V, 5)$, qui la ravagerait, produisant sa rédemption. Le Christ, au ventre de Marie, prend son envol d'oiseau de feu:

maria, nom pluriel des eaux - usage dense du sein et nativité du feu. («la Malemer»)

C'est bien cela qui manque aux hommes, à la foule des tièdes («jungle tiède", $V, 6$ ): la gloire du Feu vivant, lui-même dévoré par sa fièvre de Dieu, «gloire amoureuse d'un corps incendié".

Pour le poète, il n'est de corps de gloire que le poème; il n'est de feu que l'écriture, qui est l'alchimie non pas du verbe, mais du monde.

Rina Lasnier, dans la surcharge de l'image, est notre absence à tous. 